\title{
A Simulation Model for the Smart Irrigation System using Arduino
}

\author{
P. Mercy Nesa Rani ${ }^{1}$, A. P. S. Thengal ${ }^{2}$, Paominhao Hangsing ${ }^{3}$, L. I. P. Ray ${ }^{4}$, \\ T. Rajesh ${ }^{5}$, Kamalika Datta ${ }^{6}$ \\ National Institute of Technology - Meghalaya, Bijni Complex, Shillong, Meghalaya, India ${ }^{1,2,3,6}$ \\ College of Post Graduate Studies, Central Agricultural University, Umiam, Meghalaya, India ${ }^{4,5}$
}

\begin{abstract}
Irrigating the crop is one of the greatest challenges in agriculture. This irrigation process consumes significant amount of time, when a farmer has to irrigate multiple agriculture fields across different geographical areas. Therefore, we propose a model for the smart automated irrigation system. Initially, the environmental data such as soil moisture level, humidity and temperature have been acquired from the different sensor nodes placed across the agricultural field. Then, the microcontroller estimates the soil temperature and moisture level from the acquired data and start the irrigation process accordingly. When the irrigation process is over, the physical parameters such as humidity, temperature and volume of water irrigated can be uploaded to the cloud server. Later, the user can access the cloud-server for viewing the status of the irrigation system. This system will definitely help the farmers to effectively irrigate the agricultural field.
\end{abstract}

Keywords: Agriculture, Smart Irrigation Facility, Sensors, Crop

\section{INTRODUCTION}

India is an agricultural based country and our economy mainly depends on agriculture. Agriculture is one of the areas where water is required in tremendous quantity. Hence, irrigation plays an important role in agriculture. It influences the different crop production. In India, nearly $80 \%$ of total annual rainfall occurs in four months, i.e. from mid of June to mid of October. So it is very necessary to irrigation for farm field during the rest of the eight months. There are different types of irrigation methods viz., Sprinkler System, Drip Irrigation, Ditch Irrigation, and Terraced Irrigation which can be used to irrigate the crop.

Wastage of water is a major problem in the existing irrigation. Excess of water is given to the fields most of the time. We often neglect the importance of preserving the environment. We are utilizing the water resources without thinking how the earth will be in future with respect to water. The world population is increasing and will rise to nine billion by 2050. So, we have to think about the safety of our food which comes from agricultural products.

Smart agricultural system is one of the solutions to solve this problem. In our system, timely monitoring of soil temperature and watering the soil in such a way that it makes every single drop count i.e. minimum utilization of water resources. These methods allow year-round growth. This irrigation process consumes significant amount of time, when a farmer has to irrigate multiple agriculture fields across different geographical areas. By combining advanced computer controllers with actuators and sensors, the farmer can offer more food production and better yield. Through Smart agriculture system we can optimize water consumption as well as improve the agricultural yield.

In this paper, we present a design for the automated irrigation system. All the environmental data such as soil moisture level, humidity and temperature have been acquired from the different sensor nodes. Then, the microcontroller calculates the soil temperature and moisture level from the acquired data and start the irrigation process. Once the irrigation process is over, the physical parameters such as humidity, temperature and volume of water irrigated will be processed by microcontroller. Then, the data will be uploaded to the cloud server with the help of a GSM module.

The rest of the paper is organized as follows. Section II presents the background concepts needed for automated irrigation system. Section III proposes the method of basic irrigation system and the improved irrigation system. Section IV explains the experimental results and discussions followed by the conclusion in section V. 


\author{
Vol. 8, Issue 2, February 2019
}

\title{
2. PRELIMINARIES
}

In this section, we look into the irrigation system and the various reviews on the smart irrigation system which helps to understand the depth of this approach.

\subsection{Irrigation System}

In agriculture, most of the Wireless Sensor Network (WSN)-based applications have been developed to automate the different agricultural works. For example, WSN for environmental condition monitoring with information of soil nutrients is applied for predicting crop health and production quality over time. Irrigation scheduling is predicted with WSNs by monitoring the soil moisture and weather conditions. This scenario is depicted in Figure 1. Here, the different sensors are placed across the agricultural field. The data are acquired from the different sensors and updated in the cloud server using the gateway node. The user can obtain the various details of the crop and the corresponding soil from the cloud server.

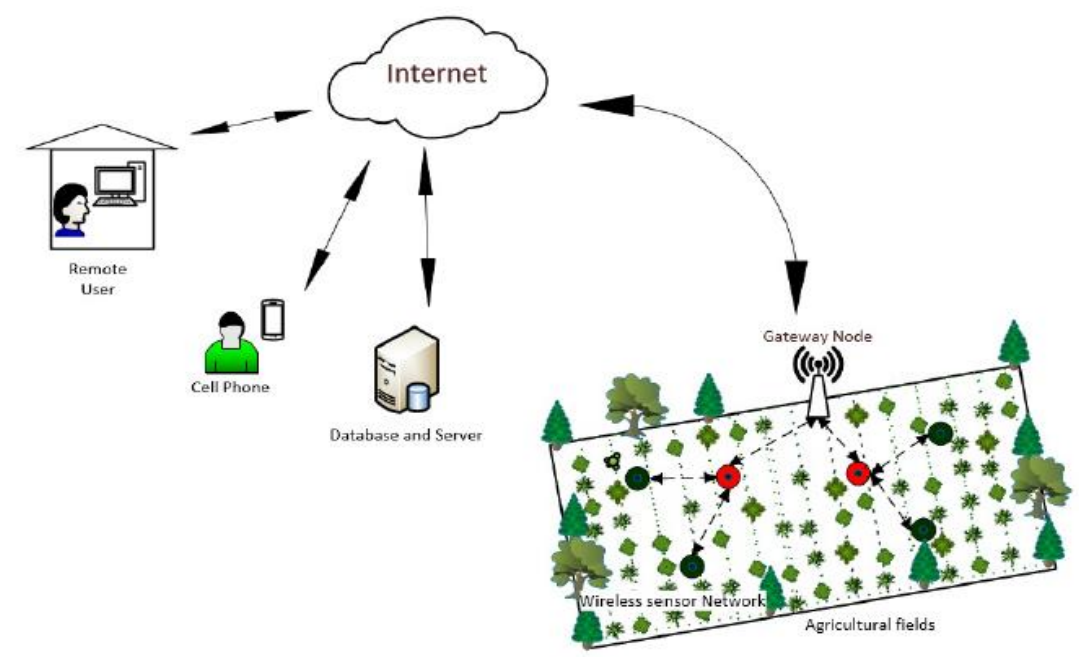

Fig 1: Deployment of WSN in Irrigation System

\subsection{Various Works on Automated Irrigation System}

The various reviews on different works of automated irrigation system has been presented in this subsection.

In [1], Sahu et al. proposed a cost effective smart irrigation system which was constructed using raspberry-pi and arduino. The raspberry-pi is used to control a three way electromagnetic valve which selects the direction of the water flow. While the valve is kept open, the motor is switched ON and the farmer is notified through Short Messaging Service (SMS) or email to his registered email account. In [2], Paucar et al. has proposed the wireless system architecture for smart irrigation. The system utilizes a wireless sensor network to gather sensor data to evaluate the environmental conditions. Riadh et al. [3] has proposed a smart irrigation system at a farm scale covering field crops as well as plantations. In [4], Sanga et al. has also discussed about the low cost smart irrigation system which enhances the farmer's life and Indian economy. Navarro et al. [5] proposed the decision support smart irrigation system. This system estimates the weekly irrigations needs of the crop and it can predict the irrigation needs of one or several crop, based on the information provided by the sensors and also from the historical archive of the previous year irrigation reports.

Arvind et al. [6] has suggested an economical and easy way to use arduino-based automated irrigation system that is remotely controlled by an android smart phone. Natta et al. [7] has developed an automated agricultural system which measures the environmental data from the vegetable crop using wireless sensor networks and controls the irrigation system. Various measured environmental data were sent back to the server node through coordinator nodes. In [8], Kumar et al. has proposed an automated irrigation system for efficient management of water resources in agricultural sector. The System uses impedance based sensors to measure the relative moisture of the soil. Nesa et al. [9] proposed the automated irrigation system using Medium Access Control (MAC) protocol through wireless sensor networks.

In [10], Patricia et al. has presented the implementation of cost effective smart irrigation system using modified wireless sensor network. Ayman et al. [11] has demonstrated the configuration of a wireless sensor network for management of crop irrigation from canals. The system consists of flow sensors, water level sensors, gateway and an irrigation management platform. The gateway sends the received data to the web server through GPRS connection. The web server is linked with database for tracking the water levels in all the canals. The advantage of the proposed system is its low cost nodes and location based water consumption mapping. Milos et al. [12] has presented an overview of smart irrigation software solutions. The presented solutions use the environmental data to make real time decisions and plan the irrigation schedule. The data were gathered from various sensors, existing databases, internet sources and 


\author{
Vol. 8, Issue 2, February 2019
}

weather stations. In [13], a fuzzy logic based irrigation system has been presented. Direct sensor data like air temperature, soil-moisture, solar radiation and water flow have been given acquired using different sensors and are given directly as the inputs to fuzzy logic controller. It requires more number of conditions for determining the time and duration of irrigation.

\title{
3. PROPOSED SYSTEM
}

In this section, we discuss about the various components used in the automated irrigation system. The following subsection proposes the implementation of basic irrigation system. The next subsection discusses an improved design of smart irrigation system.

\subsection{Components Used in Smart Irrigation System}

The different components used in the smart irrigation system have been explained as follows.

\subsubsection{Microcontroller Board: ARDUINO UNO R3}

Arduino is basically an open source electronics prototyping platform. Arduino Uno R3 is a microcontroller board consisting of two micro-controllers viz., ATMEGA 328P-PU and ATMEGA16U2. The microcontroller board has a 14 digital input/output pins and 6 analog input pins with 10 bit Analog to Digital Converter (ADC). An external programmer is not required here because the ATMEGA 328P-PU already comes with boot loader. The pictorial representation for the arduino uno is given in Figure 2(a).

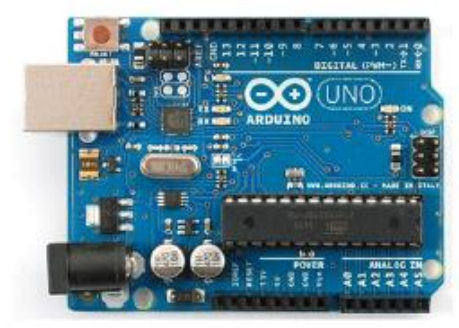

(a)

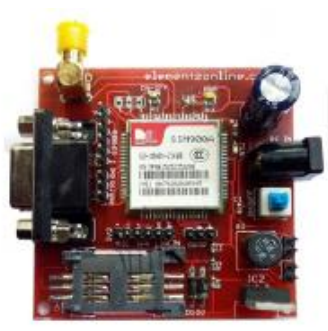

(b)

Fig.2: (a) Arduino Uno, (b) GSM Board

\subsubsection{GSM module: SIM 900A}

The Global System for Mobile Communications (GSM) module consists of a SIM900A modem and on-board RS-232 for Transistor-Transistor Logic (TTL) communication. SIM900A is a compact wireless dual band GSM/General Packet Radio Service (GPRS) module, operating on frequencies 900/1800 MHz. The baud rate is configurable from 1200115200. Both baud rate and operating frequency can be configured using AT COMMANDS. The GSM/GPRS Modem also has an internal Transmission Control Protocol/Internet Protocol (TCP/IP) stack which enables us to connect with internet via GPRS. It has a very low power consumption of $1.5 \mathrm{~mA}$ while the module is in sleep mode. The operational temperature of the module ranges from $-40 \mathrm{C}$ to $+80^{\circ} \mathrm{C}$. The on board voltage supply is $12 \mathrm{v}$. The graphical representation for the GSM module is given in Figure 2(b).

\subsubsection{Moisture Sensor}

Soil moisture sensor is sensitive to the ambient humidity. They are generally used to detect the moisture content of the soil. The sensor module can give output in both digital and analog forms. It also has an on-board LM393 comparator Integrated Chips (IC) and a potentiometer for configuring a threshold value. The analog signal can be fed to an Analog/Digital (A/D) converter for obtaining a precise sensor value. The soil moisture sensor operates from 3.1 to $4.8 \mathrm{v}$ which is represented in Figure 3(a).

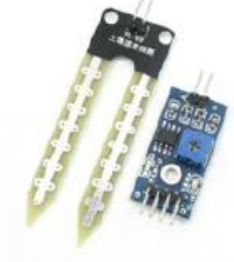

(a)

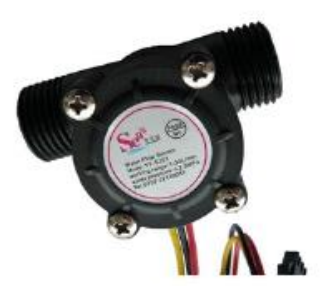

(b)

Fig.3: (a) Moisture Sensor (b) Flow Sensor 


\subsubsection{Flow Sensor}

Water flow sensor is essentially a plastic valve body with a hall-effect sensor and a water rotor. When water flows through the rotor, rotor rolls. The rotor speed changes with different rate of flow. The hall-effect sensor detects the change in rate of flow and sends the output in the corresponding pulse signal. The flow sensor can detect flow rate from 1-30 L/min. The maximum pressure rating of the sensor is $2 \mathrm{Mpa}$ which is shown in Figure $3(\mathrm{~b})$.

\subsubsection{Humidity Sensor}

The AM2302 consists of a capacitive humidity sensor and thermistor temperature sensor enclosed in a plastic body. It requires a voltage supply of 3 to $5 \mathrm{v}$ DC. The rated current consumption is $2.5 \mathrm{~mA}$. The module puts digital signals on the DATA pin every 2 seconds. Relative humidity can be sensed from 0 to $100 \%$ R.H by using this module. The accuracy in sensing humidity can be achieved up to 2 to $5 \%$ R.H. Figure 4 shows the humidity sensor.

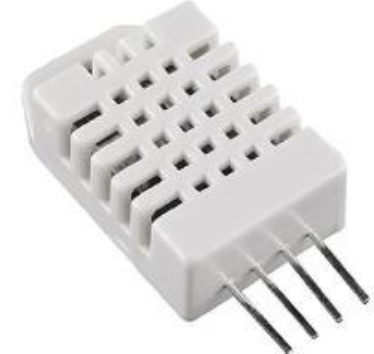

Fig.4: Humidity Sensor

\subsubsection{Water Pump}

Water pump is a submersible water pump having output power of $18 \mathrm{w}$. The operating voltage of the pump is $220 / 240 \mathrm{~V}$ AC @ $50 \mathrm{~Hz}$. The maximum flow output is $1100 \mathrm{~L} / \mathrm{H}$. The pump must be always operated totally submerged and dry runs should be avoided.

\subsubsection{Valve}

The solenoid valve is an electro-mechanical valve. Water is allowed to pass through the valve only when it is energized. It requires a constant power supply of 24-36V DC. The valve blocks the flow of water when the power supply is cut-off. In the next section, we will look into the basic irrigation system.

\section{BASIC IRRIGATION SYSTEM}

The smart irrigation system acquires the environmental data periodically from the sensors. The microcontroller estimates the soil temperature and moisture level from the acquired data. An interrupt signal is generated when the moisture levels fall below a predetermined threshold value. The signal causes the microcontroller to initiate the irrigation. To prevent the pump from running in a dry state, the water level of the reservoir is monitored. The pump is switched on only when the water level is above a certain limit. Solenoid valves are used for blocking and allowing the flow of water. Also, the flow sensor is used to monitor the flow rate of the pump.

The flow rate is used to estimate the volume of water consumed in the process. As long as there is water deficiency in the soil, the pump is kept running. Once the soil has been sufficiently irrigated, the pump is turned off and the valve is closed. The farmer is kept updated about the status of the system regularly through SMS. The environmental data and the estimated parameters are also uploaded to cloud storage.

All the environmental data such as soil moisture level, humidity and temperature is collected from different sensor nodes. The moisture sensors are connected to the analog pins of the Arduino. This allows us to sample the moisture levels only 10,000 times in a second. Also, the humidity sensor has a limitation of refreshing the output every two seconds only. The acquired data is sent collectively to the web server using Hyper Text Transfer Protocol (HTTP) service from the GSM module.

\section{AN IMPROVED SMART IRRIGATION SYSTEM}

In the basic irrigation system, we have irrigated the field with some pre-defined time periods and notified the user through SMS when the task was completed. But the system cannot monitor any environmental parameters other than the moisture level. For proper irrigation and reduction in water stress periods, we need to monitor other environmental parameters also. The moisture sensor used in this system has the capability of sensing up to $100 \mathrm{ml}$ of water. Thus, beyond this volume of water the sensor is unable to differentiate. 
Vol. 8, Issue 2, February 2019

Irrigation of crops depends on the crop characteristic and soil moisture characteristic properties. The knowledge of these characteristics is highly desirable to achieve better crop yields and irrigation efficiency. Application of this knowledge will reduce water stress among crops and directly result in more crop yield.

The block diagram of proposed irrigation system is shown in Figure 5. The microcontroller is pre-set with a default irrigation routine. When it is time to start irrigation, the microcontroller checks if the soil surrounding any node is dry. If it is so, then the microcontroller opens the valve which is blocking the water supply to the node. After opening the valve, the pump will be switched on and water will begin to flow. The system continuously checks for the dry nodes and starts watering the nodes still all the nodes become wet. Then, the information is passed to the microcontroller.

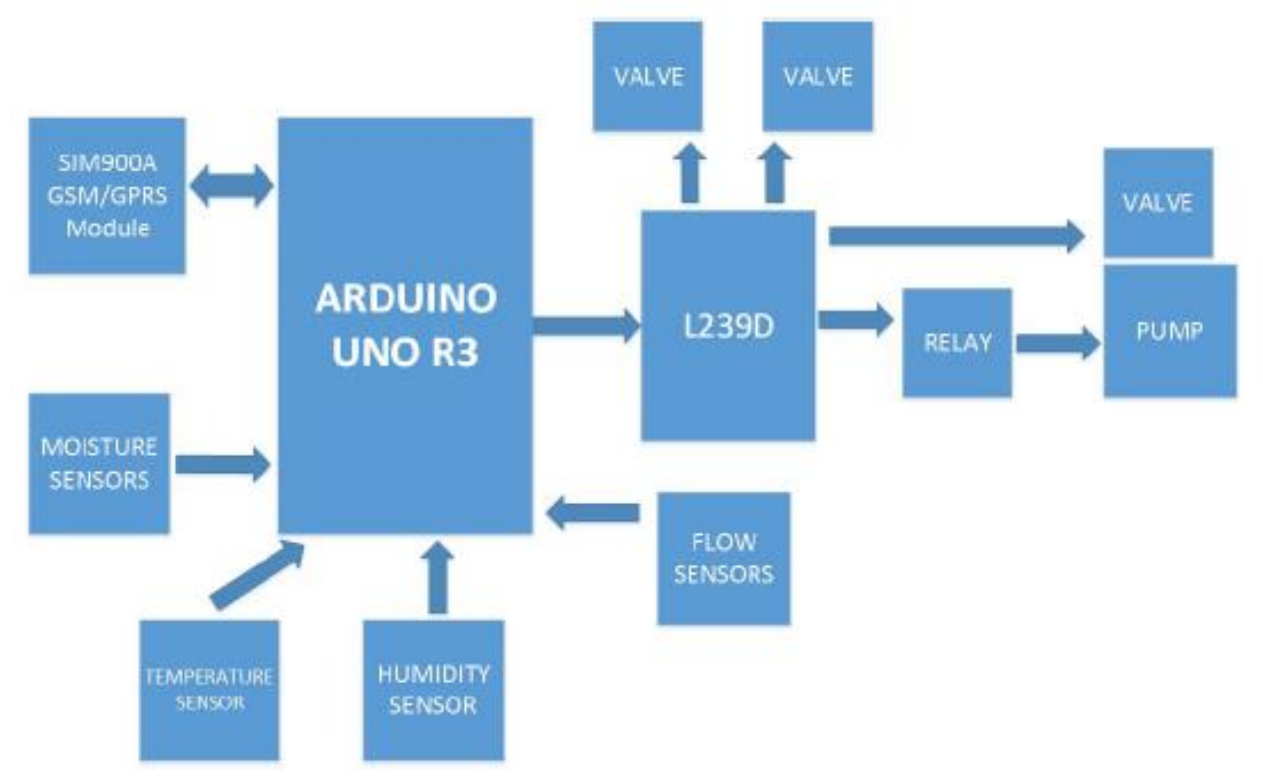

Fig.5: Block Diagram

Once the irrigation process is over, the physical parameters such as humidity, temperature and volume of water irrigated are acquired and processed by microcontroller from time to time. After acquisition, it uploads the data to the cloud server with the help of a GSM module. The GSM module is initialized by sending various AT commands through a serial communication interface. First, the GPRS connection settings are configured and then an HTTP connection is opened. An IP address is allotted to the GSM module. The microcontroller will next configure the URL string for accessing the website of the server. All the data which needs to be uploaded is put in end of the URL as "GET method" parameters. Once the entire configuration has been done properly, the system will start accessing the URL. As soon as the page is fetched completely, the GSM module stores it in its memory and notifies the microcontroller. The server extracts the data contained in the URL string and inserts the data along with date and time in the database.

Later when the user wants to access the cloud-server for viewing the status of the irrigation system, he can simply login through the mobile app. Upon logging in successfully, the dashboard pops up. From there, he can view the irrigation history log or start irrigating manually. The farmer can easily configure the system either through his mobile phone or through a web browser. Once the mobile app is installed on the smart phone, the farmer can control the irrigation system manually from anywhere. This will give them real time data access to the user and will allow them to access the entire smart irrigation system as well. After that, we have also collected the soil samples from different areas in shillong and the texture analysis is carried out which is presented in the next subsection.

\subsection{Soil Texture Analysis}

Three soil samples have been collected for soil texture analysis. The analysis is carried out for the collected samples with the help of a pressure plate extractor. After the analysis is done, the above mentioned soil samples are found to be of soil type sandy loamy soil. Sandy loamy soil is a soil type which mostly contains sandy particles. However, they do contain enough clay and silt. For sandy loamy soil, the water drains very quickly and it hardly contains micro nutrients. Frequent irrigation is required for the above mentioned soil, since it cannot hold water sufficiently. Due to micro nutrient deficiency in sandy loamy soil, it is necessary to add manures. Field capacity can be defined as the percentage of water content which remains in the soil after excess water has been drained away.

From the analysis report we have found that field capacity of the soil sample is $34.35 \%$ and the corresponding pressure is roughly 0.289 bar. Permanent wilting point can be defined as the percentage of water content in the soil beyond which the plant cannot extract any water from the soil and begins to wilt due to deficiency of water. For the analysed 


\section{Vol. 8, Issue 2, February 2019}

soil sample, its permanent wilting point is $17.63 \%$ and the corresponding pressure is 15 bar. The curve plotted with moisture content versus pressure is depicted in Figure 6. From the graph, we can conclude that as the moisture content increases, then pressure decreases. Since, the soil type is sandy loamy soil we need to irrigate the soil frequently. In order to do this, adjustments in the system is to be made in such a way that the system monitors the soil moisture content regularly and makes sure the moisture content stays between the field capacity and permanent wilting point.

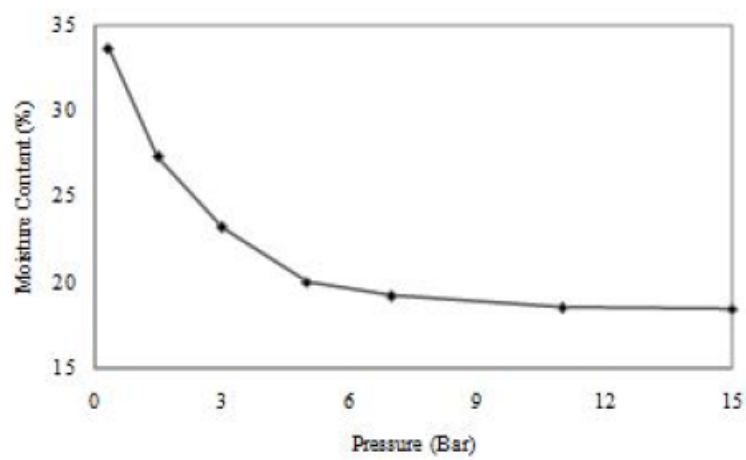

Fig.6: Texture Analysis

\section{EXPERIMENTAL RESULTS AND DISCUSSIONS}

In the basic irrigation system, initially sensors are placed in a dry soil sample in a plastic container. Then, the voltage across the sensor is measured and converted in ADC values. After that, the ADC values are measured against the different moisture level present in the soil. The values are reported in the Table 1.

Table 1.Sensor Reading Vs Moisture Level

\begin{tabular}{|c|c|}
\hline Water Level (ml) & $\begin{array}{c}\text { ADC Output Electrode } \\
\text { depth of 40mm (bit value) }\end{array}$ \\
\hline 0 & 1020 \\
\hline 25 & 814 \\
\hline 50 & 653 \\
\hline 75 & 511 \\
\hline 100 & 400 \\
\hline 125 & 377 \\
\hline 150 & 390 \\
\hline 175 & 374 \\
\hline 200 & 385 \\
\hline 225 & 395 \\
\hline 250 & 375 \\
\hline
\end{tabular}

It was observed that initially, the ADC output values change rapidly with increasing water levels. This happens because with addition of water to the soil the minerals present in it begins to dissolve. As soon as they all dissolve, the values cease to change rapidly. Water was added to the soil until the ADC values began to remain constant. From the experiment, it was observed that the voltage across the sensor electrode remained constant after a water level of $150 \mathrm{ml}$. We have fitted the curve with linear model which is shown in Figure 7.

In the improved irrigation system, the ADC values are measured for the new moisture sensor against the different moisture level present in the soil. The values are reported in the Table 2 . The ADC output values change rapidly with increasing water levels only when the soil is dry. This happens because with addition of water to the soil the minerals present in it begins to dissolve. When all the minerals get dissolved, the values cease to change rapidly. Water is added to the soil until the ADC values begin to remain constant. From the experiment, it is observed that the voltage across the sensor electrode remains constant after a water level of $200 \mathrm{ml}$. After that, we fitted the curve of linear model for the water level vs ADC values which is shown in Figure 8. 
Vol. 8, Issue 2, February 2019

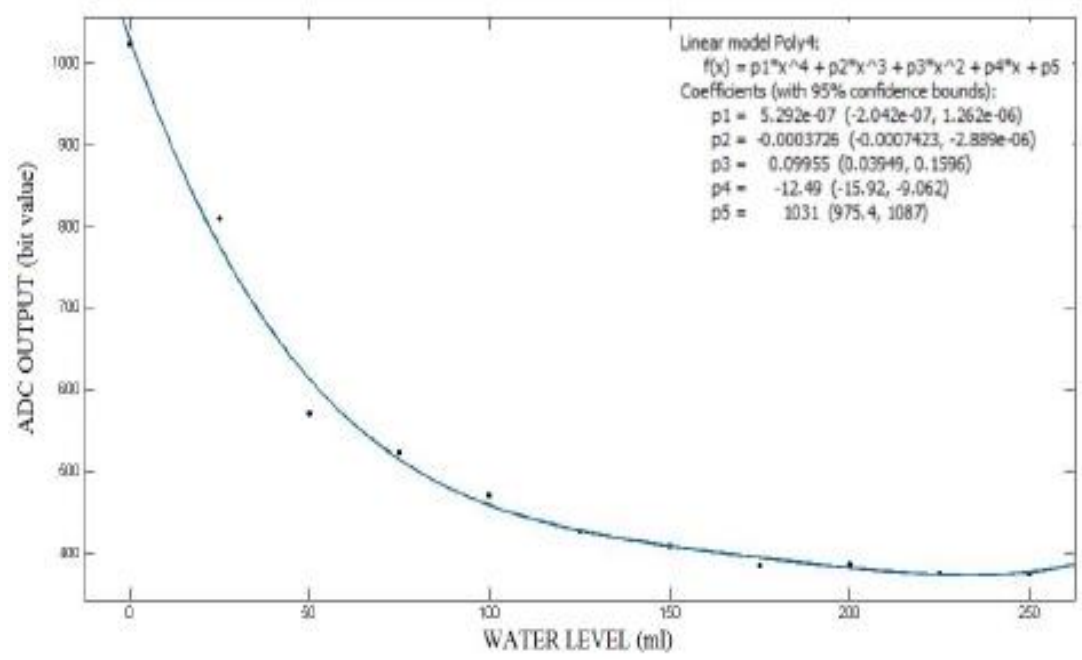

Fig.7: Water Level Plot

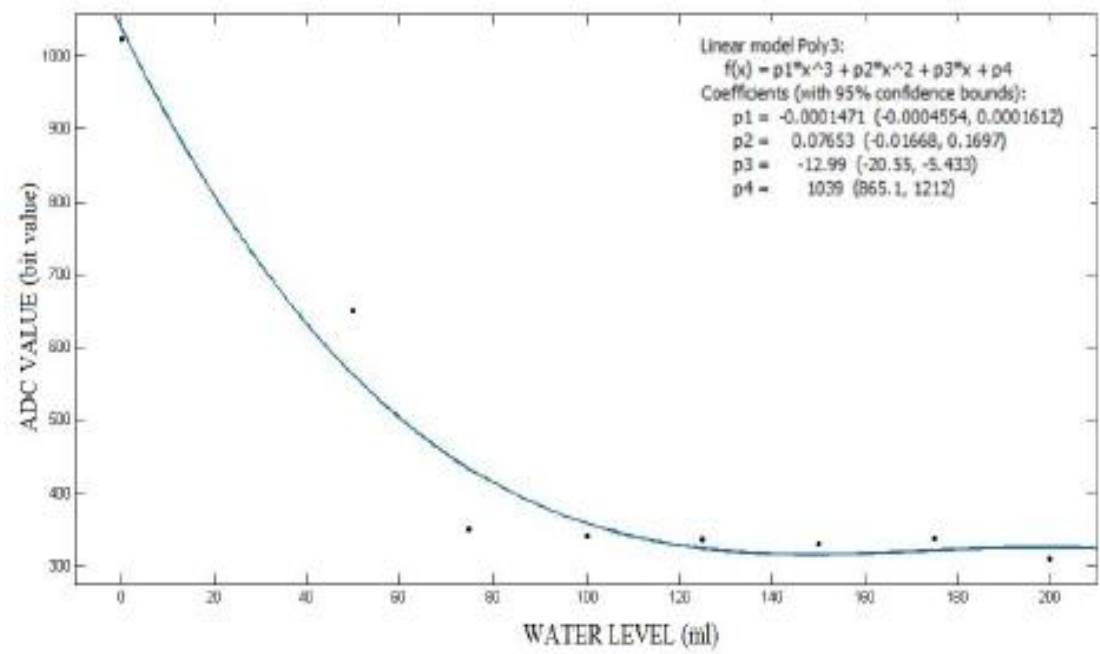

Fig.8: Water Level Plot

Table 2. New Sensor Reading Vs Moisture Level

\begin{tabular}{|c|c|}
\hline Water Level (ml) & $\begin{array}{c}\text { ADC Output Electrode depth } \\
\text { of 40mm (bit value) }\end{array}$ \\
\hline 0 & 1022 \\
\hline 25 & 810 \\
\hline 50 & 570 \\
\hline 75 & 522.5 \\
\hline 100 & 470 \\
\hline 125 & 426.75 \\
\hline 150 & 408.5 \\
\hline 175 & 384.25 \\
\hline 200 & 385 \\
\hline 225 & 375 \\
\hline 250 & 375 \\
\hline
\end{tabular}




\title{
IJARCCE
}

\section{International Journal of Advanced Research in Computer and Communication Engineering}

\author{
Vol. 8, Issue 2, February 2019
}

\section{CONCLUSION}

In this paper, we have presented the automated irrigation system which helps the farmers to irrigate optimal water to the crop without affecting its growth. This irrigation system determines the moisture content of the soil by measuring the resistivity of the electrodes which are inserted into the soil and water the plant accordingly. The moisture level of the soil is determined by measuring the ADC values of the moisture sensors. Depending on the moisture level in the soil, the water pump is switched on to irrigate the crop. The drawback of such moisture measurement technique is that it requires individual calibration and it may fails in saline soil. By increasing the length of the electrode we can increase the range of sensing the water content in the soil. In order to monitor large agricultural field, we can also deploy wireless sensor networks.

\section{REFERENCES}

[1]. C. K. Sahu and P. Behera. A low cost smart irrigation control system. In IEEE sponsored $2^{\text {nd }}$ International Conference on Electronics and Communication Systems (ICECS), pages 1146--1151. IEEE, 2015.

[2]. L. G. Paucar, A. R. Diaz, F. Viani, F. Robol, A. Polo, and A. Massa. Decision support for smart irrigation by means of wireless distributed sensors. IEEE, 2015.

[3]. R. Zaier, S. Zekri, H. Jayasuriya, A. Teirab, N. Hamza, and H. Al-Busaidi. Design and implementation of smart irrigation system for groundwater use at farm scale. In $7^{\text {th }}$ International Conference on Modelling, Identification and Control (ICMIC 2015) Sousse. IEEE, 2015.

[4]. S. Malge and K. Bhole. Novel, low cost remotely operated smart irrigation system. In International Conference on Industrial Instrumentation and Control (ICIC), pages 1501--1505. IEEE, 2015.

[5]. H. N. Helln, J. M. D. Rincon, F. S. Valles, R. D.Miguel and, and R. T. Snchez. A decision support system for managing irrigation in agriculture. Computers and Electronics in Agriculture, Pages:121--131, 2016.

[6]. A.N.Arvindan and D. Keerthika. Experimental investigation of remote control via android smart phone of arduino based automatic irrigation system using moisture sensor. In $3^{\text {rd }}$ International Conference on Electrical Energy Systems, pages 168--175. IEEE, 2016.

[7]. N. Kaewmard and S. Saiyod. Sensor data collection and irrigation control on vegetable crop using smart phone and wireless sensor networks for smart farm. In Conference on Wireless Sensors (ICWiSE), pages 106--112. IEEE, 2014.

[8]. A. Kumar, K. Kamal, M. O. Arshad, T. Vadamala, and S. Mathavan. Smart irrigation using low-cost moisture sensors and xbee-based communication. In Global Humanitarian Technology Conference, pages : 333--337. IEEE, 2014.

[9]. M. N. Sudha, Valarmathi M, and Babu A. Energy efficient data transmission in automatic irrigation system using wireless sensor networks. Computers and Electronics in Agriculture, 78(2)pages: 215--21, 2011.

[10]. P. C. Burbano, I. M. Garcia, and A. M. Arcentales. Ad-hoc network implementation and experimental testing using low cost and cots components. In International Work Conference on Bio-inspired Intelligence (IWOBI), pages: 133--137. IEEE, 2014.

[11]. A. M. Hassan. Web-based irrigation management for open canals using wireless sensor networks. In Conference on Wireless Sensors (ICWiSe2013), pages : 102--107. IEEE, 2013.

[12]. Milo Brajovi, Stefan Vujovi, and Slobodan ukanovi. An overview of smart irrigation software. In 4th Mediterranean Conference on Embedded Computing, pages: 353--356. IEEE, 2015.

[13]. Touati F, Al-Hitmi M, and Benhmed KTabish R. A fuzzy logic based irrigation system enhanced with wireless data logging applied to the state of qatar. Computers and Electronics in Agriculture, 98:233--41, 2013. 